\title{
LED-transistor optical vascular measure: Calibration, replicability, and comparability to strain-gauge measures
}

\author{
MICHAEL F. SUNDERMAN and J. RICHARD JENNINGS \\ University of Pittsburgh, Pittsburgh, Pennsylvania 15213
}

\begin{abstract}
Recently, a LED-transistor optoelectronic package (Sensor Technology 850A) has been used to measure peripheral vascular responses. This report examines the calibration and measurement replicability of this device and relates device output to flow measures derived from venous occlusion plethysmography. Measures were taken from the thumbnails of 11 male volunteers. A correlational analysis of sequential amplitude readings compared calibrated and uncalibrated values. Calibration failed to increase the sensitivity of the measures. Replicability of measurement was assessed by removing and replacing the device five times. A correlation of $.87 \mathrm{sug}$ gested that the readings are replicable. Finally, the amplitude of the optical signal was correlated to concurrent flow measures. Only modest correlations were observed as computed across time within individuals or between individuals. The optical device does not appear to be a direct measure of flow as assessed by venous occlusion plethysmography, rather, it may be heavily influenced by the mechanical motion of the vessel wall.
\end{abstract}

Blood flow is an important parameter of vascular function that is regulated by the sympathetic nervous system. A noninvasive, beat-by-beat measure of blood flow is currently unavailable, although a variety of new techniques are promising. Jennings, Tahmoush, and Redmond (1980) investigated the utility of a simple, inexpensive LED-transistor optical device as a measure of peripheral vascular reactivity. Complete descriptions of this device's electrical properties, characteristics, and applications are available elsewhere (Jennings et al., 1980; Lee, Tahmoush, \& Jennings, 1975). The previous work, as well as this paper, are attempts to refine devices previously referred to as photoplethysmographs. We prefer not to use this term as it implies that optical techniques measure volume or "fullness" directly.

Issues are examined that are important for the routine use of optical vascular measures. First, Jennings et al. (1980) describe a system of calibrating photo devices in units of power (milliwatts). In principle, this system improves the comparability of photoelectrically dissimilar devices, but the practical significance of the system for measures in man has not been addressed. Second, the replicability of measurement over different occasions using the same and different devices is explored. Measurement errors (errors of replicability) should be small relative to differences found between individuals and experimental conditions.

We would like to thank Charles Rakaczky and Donna Dow for their assistance in scoring the physiological signals and W. Winslow Westervelt for reading an initial draft of this paper. Address all reprint requests to J. Richard Jennings, Department of Psychiatry, University of Pittsburgh, 3811 O'Hara Street, Pittsburgh, Pennsylvania 15213.
A third issue is the relationship of optical measures to volumetric measures of blood flow in humans. If the optical device measures blood flow, then its output will be highly correlated with flow derived from the generally accepted venous occlusion technique. The occlusion technique momentarily stops venous return from a limb and assesses the rate of increase in volume of the limb during this time. Strain-gauge plethysmography, rather than the earlier volumetric technique, is commonly used with the venous occlusion technique. Applications and characteristics of the strain-gauge technique are widely documented (Formel \& Doyle, 1957; Greenfield, Whitney, \& Mowbray, 1963; Jennings et al., 1980; Whitney, 1953).

In the study reported here, an indium-galium alloy in rubber strain gauge is used. This device is similar to the mercury-in-rubber strain gauge, except that it overcomes some of the thermal problems inherent in the mercury device.

\section{METHOD}

\section{Subjects}

Eleven male volunteers, between 19 and 28 years of age, served as subjects. All were healthy undergraduate or graduate students at the University of Pittsburgh. Volunteers were paid $\$ 6$ for their participation in the experiment.

One of the volunteers' data were discarded because of repeated inversion of the psychometric signals. Inversion is rare and appears to be a function of vascular anatomy, device placement, and device characteristics (Jennings \& Choi, in press).

\section{Apparatus \\ A Medasonics SG-6 strain gauge was used for the volumetric strain-gauge device. The temperature coefficient of resistivity for}


this strain gauge is approximately 500 parts per million per degree centigrade. This means that temperature change has no appreciable effect on the device. The strain gauge was balanced and amplified using a laboratory-built resistance bridge and operational amplifier in conjunction with a Grass 7PIA preamplifier. A Medasonics digit occluding cuff was used for the venous occlusion.

The strain gauge was calibrated by means of a laboratorybuilt volumetric calibration device. This device consists of two glass syringes $(1.5$ and $.5 \mathrm{cc})$, a 12 -in. length of nonexpandable Tygon tubing, a 5-in. length of expandable neoprene tubing (.625 in. diameter $x .5$ in. bore), and a high-pressure control valve. The Tygon tubing was connected to the outlets of the syringes. The ends of the tubing were connected to a single length of tubing, which in turn was connected to the expandable tubing. The syringe and tubing system were filled with vacuum pump oil (see Figure 1).

The photometric device is a Sensor Technology $850 \mathrm{~A}$ infrared LED-transistor transducer. A complete description of the device, calibration methods, and device characteristics is reported elsewhere (Jennings et al., 1980). Calibration is based on varying the light output of a known infrared source and observing the phototransistor response. A parabolic function relating light input to transistor output is computed. Calibration functions are then derived by taking derivatives of the parabolic function at

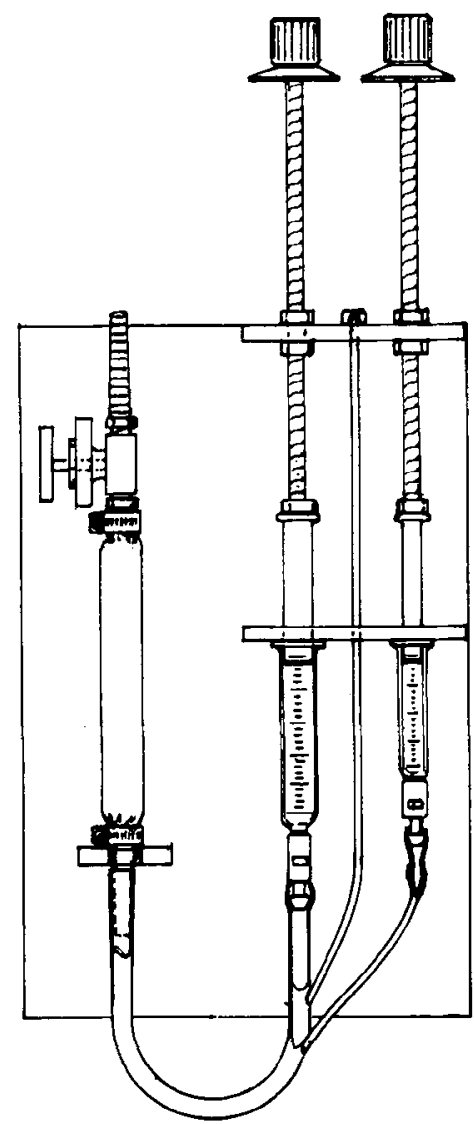

Figure 1. Volumetric strain-gauge calibration device. The strain gauge is placed around the center of the large tube (upper center). The langer syringe (center left) allows for gross volume adjustment. The small syringe (lower left) is for fine adjustments in the range of $.1 \mathrm{cc}$ to $1.5 \mathrm{cc}$. the operating point of the phototransistor once placed on the volunteer.

\section{Procedure}

The strain gauge was placed around the expandable tube of the device described above. The volume of the expandable tube was increased in increments of $.4 \mathrm{cc}$ by expelling the oil from the smaller syringe into the tubing system. The range of the volume change was from .0 to $1.6 \mathrm{cc}$. Resulting changes in the strain gauge were recorded on the polygraph. A total of 20 expansion series were performed, with the strain gauge being removed and reaffixed after each series. The data from the calibration series were averaged to determine the voltage change for each $.4 \mathrm{cc}$ of volume change. A linear equation fit to these data to form a calibration function accounted for $93 \%$ of the variance.

During the experiment, the volunteer was seated in an Industrial Acoustics sound-attenuating chamber. An adhesive polyurethane guide was attached to the thumbnail of each hand. The optical device was set into the guide and held in place with a small amount of collodion. Artifacts due to pressure of attachment were minimized because of the thumbnail site. Relative to other tissue, the thumbnail is less compressible and thus minimizes artifactural distortion of the vascular bed. Our prior attempts to use controlled pressure attachments to soft tissue had not proved reliable (Jennings et al., 1980).

A strain gauge was placed around the midsection of the index finger on the subject's left hand. The occluding cuff was placed upstream of the strain gauge on the finger. A thermistor was placed on the back of the left hand. The technique for applying the thermistor was the procedure described by Taub and School (1978). A three-lead (V5) attachment was used for the electrocardiogram. An Arteriosonde blood pressure cuff was placed around the right arm and connected to a Roche Arteriosonde doppler blood pressure device.

When all of the devices had been attached, the subject's chair was placed in a partially reclining position and his left hand was placed on a pad to raise it to the same level as the heart. The elevation of the hand to heart level allows for good venous return and flow (Whitney, 1953).

Physiological data were recorded during five 4-min intervals. During each interval, the strain-gauge blood flow volume was recorded twice, the electrocardiogram and optical signals were continually recorded, the volunteer's blood pressure was recorded once, and the skin temperature was recorded twice. At the end of each recording interval, the optical devices were removed and reattached to the opposite hand. Also, the strain gauge was removed and then replaced.

During the fifth recording period, the volunteer squeezed a hand dynamometer with his dominant hand, inducing an increase in blood pressure and heart rate. Iust after the dynamometer was squeezed, the strain-gauge reading was recorded during the time of reactive hyperemia (Hollander \& Bouman, 1975). Following the squeeze task, a Valsalva maneuver was performed (Korner, Tonkin, \& Uther, 1976). The strain-gauge data were recorded during the maneuver.

\section{Data Reduction}

The amplitude of every beat during venous occlusion was read from the polygraph record of the optical signal. In addition, base values corresponding to "pulse volume" were measured during each placement with a digital voltmeter. These values are the dc offset on the phatotransistor caused by the average level of light flux. The slope of the curve from the strain-gauge record was determined and analyzed for the same period. Two raters independently determined the slope by drawing a line tangent to the point corresponding to the point of maximal cuff inflation and to at least three of the pulsatile waveforms on the occlusion tracing (Greenfield et al., 1963). The skin tem- 
perature and the chamber temperature were reviewed to insure that constant conditions had been achieved.

\section{RESULTS}

\section{Optical Reliability and Calibration}

The purpose of the multiple attachment of two optical devices to the volunteers was to estimate the reliability of the instrument in estimating an individual's pulse amplitude and level of backscattered light from the finger. Essentially, the amount of variance due to methods is compared to that due to individual differences. By using two devices, we compounded typical errors due to attachment and unadjusted electronic differences between devices. Bartko (1966) has developed an intraclass correlation that we have applied to estimate the reliability of individual differences relative to total experimental error and between-device error. This approach results in a correlation that can be interpreted analogously to the between-judge reliability coefficient used in psychological testing. The same approach was also used to evaluate whether event-related responses (rather than individuals) are reliably estimated by the optical devices.

The computation of the intraclass correlation employs an analysis of variance design. This analysis has individuals (or responses) and devices as random effects. A third, fixed factor, time, was added to remove any consistent trends from the data. Table 1 shows the resulting intraclass correlations. The first row presents

Table 1

Intraclass Correlation for Calibrated and Uncalibrated Devices

\begin{tabular}{lccc} 
& \multicolumn{2}{c}{ Individuals } & \multicolumn{1}{c}{$\begin{array}{c}\text { Response } \\
\text { Pulse }\end{array}$} \\
\cline { 2 - 4 } & $\begin{array}{c}\text { Pulse } \\
\text { Amplitude }\end{array}$ & Base & Amplitude \\
\hline Calibrated (Microwatts) & .85 & .26 & .81 \\
Uncalibrated (Millivolts) & .87 & .25 & .79 \\
\hline
\end{tabular}

Table 2

Photo Strain-Gauge Correlations and Means

\begin{tabular}{ccccc}
\hline \multirow{2}{*}{$\begin{array}{c}\text { Subject } \\
\text { Number }\end{array}$} & \multicolumn{4}{c}{ Measure } \\
\cline { 2 - 5 } & 1 & 2 & 3 & \multicolumn{1}{c}{4} \\
\hline 1 & .340 & .153 & 2.79 & 4.20 \\
2 & .987 & .192 & 1.89 & 2.37 \\
3 & .610 & .341 & 1.72 & 6.35 \\
4 & .878 & .176 & 2.17 & 3.32 \\
5 & .898 & -.288 & 2.38 & 9.70 \\
6 & .716 & .589 & 4.70 & 9.61 \\
7 & .963 & .720 & 1.95 & 3.74 \\
8 & .655 & .440 & 6.04 & 9.50 \\
9 & .941 & .797 & 2.71 & 14.67 \\
10 & .879 & .007 & 2.69 & 5.65 \\
\hline
\end{tabular}

Note-Measure 1 = venous occlusion rater correlation; Measure 2 = optical device amplitude vs. occlusion flow rate; Measure $3=$ mean flow rate (in milliliters of blood per $100 \mathrm{ml}$ of tissue per minute): Measure $4=$ mean optical device amplitude (in microwatts). the values when the data are adjusted by the calibration procedure, and the second presents unadjusted values. If calibration improves the comparability of devices, the intraclass correlation should be higher for the calibrated values.

Examination of the values for the estimates of individual average level of pulse amplitude shows a reasonable reliability that is not improved by calibration. In contrast, the reliability of the base values (frequently termed pulse volume) is unacceptably low. The correlations for response (right-hand column) were reasonably high, but, again, not significantly altered by calibration. The response estimates were computed entering the Valsalva and handgrip maneuvers as two levels of a response factor. The intraclass correlation thus represents the reliability of the two devices in detecting the differences between vasoconstrictive response during the Valsalva manuever and the dilative after effect of the handgrip manipulation. In short, the results show an acceptable reliability in estimating pulse amplitude and pulse amplitude responses, but not base levels. Thus between-device and replacement base levels are not reliably estimated by our technique.

\section{Strain Gauge to Optical Relationships}

Table 2 contains the results for the interrater reliability of the venous occlusion slope measures, the correlation of optical amplitude and blood flow estimated from the slope, and mean individual values for venous occlusion derived from flow and optical pulse amplitude. Slope assignment is a judgmental procedure. The average correlation of .79 shows that this judgment can be made reliably, but the individual values show at least one instance in which reliable readings were not obtained. For the purpose of further analysis, the slope values, obtained by the two raters, were averaged. These values were converted to milliliters of change per unit of time using the calibration equation developed for the strain gauge. Measurements of tissue girth and gauge width were then used to compute tissue volume. The values could then be expressed as milliliters of flow per $100 \mathrm{ml}$ of tissue per minute. The observed values are presented in Column 3 of Table 2 . The mean value of flow $(2.90 \mathrm{ml} / 100 \mathrm{ml} / \mathrm{min})$ is in the range generally reported for the finger (Burton, 1972).

Photo pulse amplitudes corresponding to the venous occlusion flow values are shown in the last column. These values, with a mean of 6.9 microW, are reasonably typical of what we have previously experienced. Mean optical and strain-gauge readings were correlated at .45 over the 10 volunteers. Photostrain-gauge correlations were also computed over measurements within volunteers. These values are tabled in the second column and yield a mean (with appropriate $r$ to $\mathrm{Z}$ transformations) of .36 . In summary, both within- and acrossvolunteer correlations show a modest correlation between optical pulse amplitude and blood flow as estimated by the venous occlusion technique. 


\section{DISCUSSION}

The reliability. estimates support the validity of using an optical technique to estimate (1) the average pulse amplitude of an individual and (2) responses of different individuals on different occasions. The technique is dependent on careful placement, with the pressure of the device on the vascular bed controlled. The acceptable but not high levels of reliability of the technique reflect in part the reduction of reliability due to unavoidable variability in placement and attachment. With appropriate care, however, optical techniques can be used to compare different individuals and occasions.

The failure of the calibration techniques to improve reliability was disappointing. Two reasons for this failure may be important. First, the devices used were not highly dissimilar electrically. The devices were chosen to be the most dissimilar of the devices delivered to us in one order from the manufacturer. Devices from different "batches" might well be more dissimilar and thus require calibration. Second, and more important, the calibration technique is based on the base-level measurements taken from the voluntecr. The results show that this measure is quite unreliable. This unreliability is likely to substantially reduce the accuracy of the calibration technique. Further development of the calibration techniques seems dependent on increasing reliability of the base-level measurements.

Venous occlusion-derived blood flow and the amplitude of the optical device signal were only moderately correlated. Prior in vitro testing has shown that the optical device is capable of sensing blood flow (Jennings et al., 1980), but the physiological application adds a number of complicating factors. For example, the elasticity of the vessel, its wall characteristics, and the properties of nonvascular tissue could all be factors. Recent work (Jennings \& Choi, in press) with in vitro and animal studies suggests that tissue movement induced by the pulse may be the primary source of the optical signal. Such movement is only partially determined by blood flow (MacDonald, 1974). This partial dependency is consistent with the .3 to .4 level of correlation observed between flow and the optical signal amplitude. More information is needed, however, before concluding that the optical signal is a function of this single source.

In summary, we have assessed the reliability of the optical measures of vascular activity over different occasions and individuals. This reliability was adequate for discriminating between individuals and between taskinduced responses. Correlation with venous occlusionderived flow indicated that this technique shared only modest variance with the optical techniques. The data suggest that the optical technique does not directly measure blood flow in humans.

\section{REFERENCES}

BARTKo, J. J. The intraclass correlation coefficient as a measure of reliability. Psychological Reparts, 1966, 19, 3-11.

Burton, A. Physiology and biophysics of the circulation. Chicago: Yearbook Medical Publishers, 1972.

Formet, P. F., \& Doyle, J. T. Rationale of venous occlusion plethysmyography. Circulation Research, 1957, 5, 354-356.

Greentield, A. D. M., Whitney, R. J., \& Mowbray, J. F. Methods for the investigation of peripheral blood flow. British Medical Bulletin, 1963, 19, 97-108.

Hollander, A. P., \& Bouman, L. N. Cardiac acceleration in man elicited by a muscle-heart reflex. Journal of Applied Physiology, 1975, 38, 272-278.

Jenninas, J. R., \& Chor, S. An arterial to peripheral puise velocity measure. Psychophysiology, in press.

Jennings, J. R., TAhmoush, A. J., \& ReDmond, D. P. Techniques in psychophysiology. In I. Martin \& P. H. Venables (Eds.), Non-invasive measurement of peripheral vascular activity. New York: Wiley, 1980.

Konner, A. M., Tonkin, A. M., \& UTher, J. B. Reflex and mechanical circulatory effects of graded Valsalva maneuvers in normal men. Journal of Applied Physiology, 1976, 40, 434-440.

LeE, A. L., TAhmoush, A. J., \& Jennings, J. R. An LEDtransistor plethysmograph. IEEE Transactions on Biomedical Engineering, 1975, 22, 248-250.

MacDonald, D. A. Blood flow in arteries. London: Edward Arnold, 1974.

Taub, E., \& School, P. J. Some methodological considerations in thermal biofeedback training. Behavior Research Methods Instrumentation, 1978, 10, 617-622.

WhitNeY, R. J. The measurement of volume change in human limbs. Journal of Physiology, 1953, 121, 1-27.

(Received for publication July 13, 1982; revision accepted October 28, 1982.) 